$5-1-2008$

\title{
Confidence Intervals for the Squared Multiple Semipartial Correlation Coefficient
}

James Algina

University of Florida, algina@ufl.edu

H. J. Keselman

University of Manitoba, kesel@ms.umanitoba.ca

Randall D. Penfield

University of Miami, penfield@miami.edu

Follow this and additional works at: http://digitalcommons.wayne.edu/jmasm

Part of the Applied Statistics Commons, Social and Behavioral Sciences Commons, and the Statistical Theory Commons

\section{Recommended Citation}

Algina, James; Keselman, H. J.; and Penfield, Randall D. (2008) "Confidence Intervals for the Squared Multiple Semipartial Correlation Coefficient," Journal of Modern Applied Statistical Methods: Vol. 7 : Iss. 1 , Article 2.

DOI: $10.22237 /$ jmasm/1209614460

Available at: http://digitalcommons.wayne.edu/jmasm/vol7/iss1/2

This Invited Article is brought to you for free and open access by the Open Access Journals at DigitalCommons@WayneState. It has been accepted for inclusion in Journal of Modern Applied Statistical Methods by an authorized editor of DigitalCommons@WayneState. 


\section{INVITED ARTICLES}

\section{Confidence Intervals for the Squared Multiple Semipartial Correlation Coefficient}

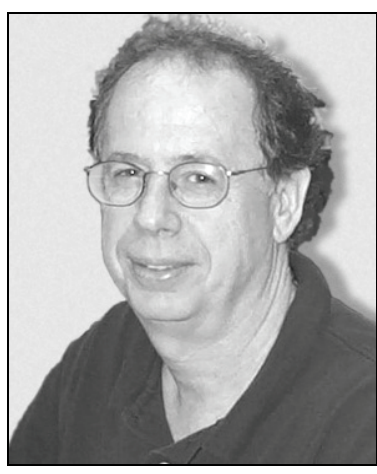

James Algina University of Florida

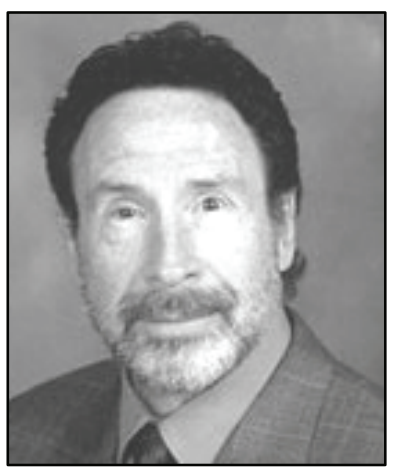

H. J. Keselman University of Manitoba

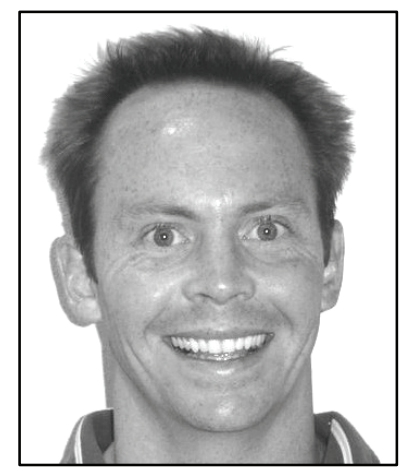

Randall D. Penfield University of Miami

The squared multiple semipartial correlation coefficient is the increase in the squared multiple correlation coefficient that occurs when two or more predictors are added to a multiple regression model. Coverage probability was investigated for two variations of each of three methods for setting confidence intervals for the population squared multiple semipartial correlation coefficient. Results indicated that the procedure that provides coverage probability in the $[.925, .975]$ interval for a $95 \%$ confidence interval depends primarily on the number of added predictors. Guidelines for selecting a procedure are presented.

Key words: Squared multiple semipartial correlation; effect size; asymptotic and bootstrap confidence intervals.

\section{Introduction}

A commonly used effect size (ES) in multiple regression analysis is the increase in $R^{2}$ when

James Algina (algina@ufl.edu) is a Professor of Research and Evaluation Methodology. His research interests are in applied statistics and psychometrics. Email him at algina@ufl.edu. H. J. Keselman is a Professor of Psychology. His research interests are in applied statistics. Email him at kesel@ms.umanitoba.ca. Randall D. Penfield is an Associate Professor of Education. His research interests are in educational measurement and psychometrics. Email him at penfield@miami.edu. one independent variable $\left(X_{j}\right)$ is added to the model. This ES, which is called the squared semipartial correlation coefficient, and is often symbolized by $\Delta R^{2}$, measures the strength of relationship between $X_{j}$ and the dependent variable $(Y)$, controlling for the other independent variables in the model. This coefficient $\Delta R^{2}$ can also be used when several variables are added to the model. In this context, $\Delta R^{2}$ is called the squared multiple semipartial correlation coefficient (Pedhazur, 1997) or the semipartial $R^{2}$ (Cohen, Cohen, West, \& Aiken, 2003) and measures the strength of association between $Y$ and the added independent 
variables, controlling for the other independent variables in the model.

Hedges and Olkin (1981) presented methods for calculating the asymptotic sampling covariance matrix for commonality components (See also Mood, 1969, 1971). These results can be used to construct a confidence interval (CI) for $\Delta \rho^{2}$, the population ES estimated by $\Delta R^{2}$. Olkin and Finn (1995) presented a method equivalent to the Hedges and Olkin method, and illustrated the new method for the case in which there is one independent variable in the model in addition to $X_{j}$ (i.e., for the case of two independent variables). Alf and Graf (1999) simplified the method and showed how to apply it in the general case of $p$ predictors, and Graf and Alf (1999) developed a computer program that computes the CI. Algina and Moulder (2001) found that when the squared semipartial correlation coefficient is of interest, researchers would need very large samples sizes $(n)$ to achieve adequate coverage probability for $\Delta \rho^{2}$. Algina, Keselman, and Penfield (2007) found that it was possible to obtain much better coverage probability, with smaller sample sizes, if percentile bootstrapping methods were used for setting CIs for the squared semipartial correlation coefficient, rather than relying on the asymptotic intervals. The purpose of the present paper was to investigate whether asymptotic or percentile bootstrap intervals would result in adequate coverage probability for $\Delta \rho^{2}$ when a squared multiple semipartial correlation coefficient is of interest.

\section{Method}

Coverage probability was estimated for the asymptotic and two percentile bootstrap CIs. Specifically, simulation was used to estimate coverage probability for combinations of $p, k, n$, $\rho_{f}^{2}$, and $\rho_{r}^{2}$, where $\rho_{f}^{2}$ is the population squared multiple correlation coefficient for a model with $p$ predictors (the full model), and $\rho_{r}^{2}$ is the population squared multiple correlation coefficient for a model with $k$ predictors (the reduced model) that are a proper subset of the $p$ predictors. In the conditions we planned to investigate, the number of predictors in the full model ranged from $p=3$ to $p=9$ in steps of 2 .

After reviewing the results we added conditions with $p=8$ predictors in the full model. The difference in the number of predictors in the full and reduced models (i.e., $p-k$ ) ranged from 2 to $p-1$ in steps of 1 . The squared multiple correlation coefficient for the predictors in the reduced model ranged from .00 to .50 in steps of .10. The squared multiple correlation coefficient for the full model ranged from $\rho_{r}^{2}$ to $\rho_{r}^{2}+.10$ in steps of .01 and from $\rho_{r}^{2}+.10$ to $\rho_{r}^{2}+.20$ in steps of .05. Sample size ranged from 50 to 200 in steps of 50. The predictors and the dependent variable were distributed as a multivariate normal distribution.

Each of the 7176 combinations of $p, k$, $n, \rho_{r}^{2}$, and $\rho_{f}^{2}$ was replicated 5000 times in order to estimate coverage probability. For each replication, six 95\% CIs were constructed: two CIs was constructed by using two variations of (a) the asymptotic method; (b) bootstrapping $\Delta R^{2}=R_{f}^{2}-R_{r}^{2} ; \quad$ and (c) bootstrapping $\Delta R_{c}^{2}=R_{f, c}^{2}-R_{r, c}^{2}$, the difference in corrected values of $R^{2}$, where

$$
R_{f, c}^{2}=R_{f}^{2}-\frac{p}{n-p-1}\left(1-R_{f}^{2}\right),
$$

and

$$
R_{r, c}^{2}=R_{r}^{2}-\frac{k}{n-k-1}\left(1-R_{r}^{2}\right)
$$

For each $\mathrm{CI}$, the proportion of the replications that contained $\Delta \rho^{2}=\rho_{f}^{2}-\rho_{r}^{2}$ estimated the probability of coverage.

Under multivariate normality of the predictors and criterion, the asymptotic variance of $R_{f}^{2}$ is

$$
\psi_{f}^{2}=\frac{4 \rho_{f}^{2}\left(1-\rho_{f}^{2}\right)^{2}}{n}
$$

(Stuart, Ord, \& Arnold, 1999). The asymptotic variance of $R_{r}^{2}$ is obtained by substituting $r$ for $f$ in the subscripting. According to Alf and Graf 


\section{I. FOR SQUARED MULTIPLE SEMIPARTIAL CORRELATION}

(1999), the asymptotic covariance between $R_{r}^{2}$ and $R_{f}^{2}$ is

$$
\begin{aligned}
& \psi_{f r}=\frac{4 \rho_{f} \rho_{r}\left[.5\left(2 \rho_{r} / \rho_{f}-\rho_{f} \rho_{r}\right)\right.}{n} \times \\
& \frac{\left.\left(1-\rho_{f}^{2}-\rho_{r}^{2}-\rho_{r}^{2} / \rho_{f}^{2}\right)+\rho_{r}^{3} / \rho_{f}^{3}\right]}{n} .
\end{aligned} .
$$

The asymptotic variance of $\Delta R^{2}$ is

$$
\sigma_{\infty}^{2}=\psi_{f}^{2}+\psi_{r}^{2}-2 \psi_{f r}
$$

and an asymptotically correct $100(1-\alpha) \%$ CI for $\Delta \rho^{2}$ is

$$
\Delta R^{2} \pm z_{\alpha / 2} \sigma_{\infty}
$$

where $z_{\alpha / 2}$ is a $z$ critical value (Alf \& Graf, 1999). In practice, the asymptotic variance is estimated by substituting $R_{f}^{2}$ and $R_{r}^{2}$ for $\rho_{f}^{2}$ and $\rho_{r}^{2}$, respectively.

Initial results indicated that in some conditions in which $\rho_{f}^{2}=\rho_{r}^{2}$, the asymptotic CI resulted in coverage probabilities above .99 . To address this problem, the lower limit of the asymptotic CI was modified. Specifically, if the lower limit of the traditional asymptotic CI was less than or equal to zero, but the $F$ test of $H_{0}: \Delta \rho^{2}=0$ was significant, the lower limit was set to a small value larger than zero. In our simulations the lower limit was set equal to .001 .

To apply the traditional percentile bootstrap, as described in Wilcox (2003), to $\Delta R^{2}$ the following steps were completed, with the first two steps completed $B$ times.

1. A sample of size $n$ was randomly selected with replacement from the simulated participants.

2. $\Delta R^{2}$ was calculated for the sample drawn in step1.

3. Once the $B$ values of $\Delta R^{2}$ were obtained, they were ranked from low to high. The lower limit of the $100(1-\alpha) \% \quad$ CI for $\Delta \rho^{2} \quad$ was determined by finding the $[\langle B(\alpha / 2)\rangle+1]^{\text {th }}$ estimate in the rank order, where $\langle B(\alpha / 2)\rangle$ indicates rounding $B(\alpha / 2)$ to the nearest whole number; the upper limit was determined by finding the $[B-\langle B(\alpha / 2)\rangle]^{\text {th }}$ estimate in the rank order.

In all conditions $B=1000$; thus, the bootstrap lower limit was the $26^{\text {th }}$ value and the upper limit was the $975^{\text {th }}$ value in the traditional percentile bootstrap.

A problem with applying the traditional percentile bootstrap to $\Delta R^{2}$ will occur when $\Delta \rho^{2}=0$, that is when $\rho_{f}^{2}=\rho_{r}^{2}$. Because $R_{f}^{2}$ will infrequently equal $R_{r}^{2}$, the CI will almost never contain 0 and the estimated coverage probability will be near zero. To address this problem, we modified the traditional percentile bootstrap by incorporating the $F$ test of $H_{0}: \Delta \rho^{2}=0$. When the $F$ test was not significant, the lower limit of the CI was set equal to zero; otherwise, the lower limit was determined by using the traditional percentile bootstrap.

To apply the traditional percentile bootstrap to $\Delta R_{c}^{2}$, steps 1 to 3 were applied with $\Delta R_{c}^{2}$ replacing $\Delta R^{2}$. Initial results indicated that in some conditions in which $\rho_{f}^{2}=\rho_{r}^{2}$, this procedure resulted in coverage probabilities above .99. To address this problem, the lower limit of the bootstrap CI was modified. Specifically, if the lower limit of the traditional CI was less than or equal to zero, but the $F$ test of $H_{0}: \Delta \rho^{2}=0$ was significant, the lower limit was set to a small value larger than zero. In our simulations the lower limit was set equal to .001 . A Visual Basic 6.0 program that computes the traditional and modified percentile bootstrap CIs for $\Delta \rho^{2}$ is available at http://plaza.ufl.edu/algina/index.programs.html The multiple regression model is $Y=\alpha+\beta_{1} X_{1}+\beta_{2} X_{2}+\cdots+\beta_{p} X_{p}+\varepsilon$. There is no loss in generality if $\alpha=0$ and/or if the variances of the dependent variable and of the independent variables are set equal to 1.0. 
According to Browne (1969, 1975), given any set of predictors that has a squared multiple correlation coefficient of $\rho^{2}$ with $Y$, it is always possible to transform the predictors so that (a) the independent variables are mutually uncorrelated and (b) the regression coefficients are equal to any set of values such that

$$
\sum_{j=1}^{p} \beta_{j}^{2}=\sigma_{y}^{2} \rho^{2}
$$

Therefore, in the simulations (a) all variables had variance equal to one, (b) the independent variables were mutually uncorrelated, and (c) $\beta_{1}=\cdots=\beta_{k-1}=0, \beta_{k}=\rho_{r}, \quad \beta_{k+1}=\cdots=\beta_{p-1}=0$, and $\beta_{p}=\sqrt{\rho_{f}^{2}-\rho_{r}^{2}}$.

Thus, the squared multiple correlation coefficient was $\rho_{r}^{2}$ for variables $X_{1}$ to $X_{k}$ and $\rho_{f}^{2}$ for variables $X_{1}$ to $X_{p}$.

The data were simulated by using the following steps.

1. Generate an $n \times p$ matrix of random variables. Each of the $p$ variables was normally distributed with mean zero and standard deviation one. All $n p$ scores were generated to be statistically independent. This matrix is $\mathbf{X}$, the matrix of scores on the independent variables.

2. Generate an $n \times 1$ vector of normally distributed random variables with mean zero and standard deviation one. All $n$ scores were generated to be statistically independent and to be independent of the scores in $\mathbf{X}$. Multiply the generated vector by $\sqrt{1-\rho_{f}^{2}}$. The resulting vector is $\boldsymbol{\varepsilon}$, the vector of residuals.

3. Construct the $p \times 1$ vector $\boldsymbol{\beta}$ in which elements 1 to $k-1$ are zero and the next element is $\rho_{r}$, the elements $k+1$ to $p-1$ are zero, and the last element is $\sqrt{\rho_{f}^{2}-\rho_{r}^{2}}$.

4. Calculate $n \times 1$ scores on the dependent variable by using $\mathbf{y}=\mathbf{X} \boldsymbol{\beta}+\boldsymbol{\varepsilon}$.

\section{Results}

The traditional bootstrap CI using $\Delta R^{2}$ was modified by setting the lower limit to zero if the $F$ test of $H_{0}: \Delta \rho^{2}=0$ was not significant; otherwise, the lower limit was determined by using the traditional percentile bootstrap. Although the modification was designed to improve performance when $\Delta \rho^{2}$ was zero, the modification could affect coverage probability of the modified CI when $\Delta \rho^{2}$ was zero or larger. Thus, it was important to determine if coverage probability of the bootstrap CI using $\Delta R^{2}$ was affected by the modification when $\Delta \rho^{2}$ was not equal to zero. To do this, we focused on the conditions in which $\Delta \rho^{2}>0$. When $\Delta \rho^{2}>0$, for each combination of $p$, $p-k$, and $n$, there are 72 combinations of $\rho_{r}^{2}$ and $\rho_{f}^{2}$. For each combination we tabulated the number of times that the estimated coverage probability was in the interval $[.925, .975]$ for the traditional and modified versions of the bootstrap CI using $\Delta R^{2}$. Results indicated that the modification did not reduce the number of conditions in which the interval contained the estimated coverage probability. The asymptotic $\mathrm{CI}$ and traditional bootstrap CI using $\Delta R_{c}^{2}$ were modified by changing the lower limit to .001 when the traditional lower limit was less or equal to zero and the $F$ test of $H_{0}: \Delta \rho^{2}=0$ was significant. For our values of $\rho_{r}^{2}$ and $\rho_{f}^{2}$, this modification could only affect the performance of these CIs when $\Delta \rho^{2}$ was zero (and in general could only affect the CI if $\left.\Delta \rho^{2}<.001\right)$. Therefore, the following results describe performance of the modified CIs.

For each combination of $p, p-k$, and $n$, we tabulated the number of times out of 78 possible combinations of $\rho_{r}^{2}$ and $\rho_{f}^{2}$ in which the estimated coverage probability was in the interval $[.925, .975]$ for the modified version of the CIs. The results are presented in Table 1. For each combination, the bold value indicates the 


\section{I. FOR SQUARED MULTIPLE SEMIPARTIAL CORRELATION}

method(s) that best controlled probability coverage. Ties between methods are indicated by an underlined bolded result. For $p-k=2$, there were combinations of $p$ and $n$ in which the bootstrap CI using $\Delta R^{2}$ performed as well or better than the other CIs. However, for other values of $p-k$, either the asymptotic or bootstrap CI using $\Delta R_{c}^{2}$ performed better than the bootstrap CI using $\Delta R^{2}$. The relative performance of the modified asymptotic bootstrap and the modified percentile bootstrap using $\Delta R_{c}^{2}$ depended on $p-k$. When $p-k=3$, the modified percentile bootstrap tended to work better if $p \leq 7$; otherwise, the two procedures worked about equally well. The modified percentile bootstrap tended to work better when $p-k$ was between 4 or 5 . When $p-k=6$, the two procedures worked about equally well, particularly when the sample size was at least 150. For $p-k$ larger than six, a value that could only occur in our design with 8 or 9 predictors in the full model, the modified asymptotic bootstrap had better control of coverage probability.

Inspection of the results suggests that for $p-k=2$, the relative performance of the modified asymptotic $\mathrm{CI}$ and the modified bootstrap CI using $\Delta R^{2}$ depends on $\Delta \rho^{2}$. The results also suggest that larger sample sizes are required to achieve control of coverage probability when $\Delta \rho^{2}$ is small. To illustrate these effects, we tabulated (Table 2) the number of times out of the 18 possible combinations of $\rho_{r}^{2}$ and $\rho_{f}^{2}$ for $\Delta \rho^{2} \leq .02$ and the number of times out of the 60 possible combinations of $\rho_{r}^{2}$ and $\rho_{f}^{2}$ for $\Delta \rho^{2} \geq .03$ (Table 3), that the estimated coverage probability was in the interval $[.925, .975]$. When $\Delta \rho^{2} \leq .02$ and $p-k=2$, neither the modified bootstrap CI using $\Delta R^{2}$ nor the modified asymptotic CI was consistently more effective than the other over all values of $p$. Temporarily defining effective as all estimated coverage probabilities within the interval $[.925, .975]$, the modified bootstrap CI using $\Delta R^{2}$ was effective at a smaller size than was the asymptotic CI (See Table 3) for $\Delta \rho^{2} \geq .03$ and $p-k=2$. In regard to sample size required to achieve good control of coverage probability, the following comments apply to all combinations of $p$ and $p-k$, with the exception of conditions in which there were nine predictors in the full model and no more than two in the reduced model. When $\Delta \rho^{2} \leq .02$, the sample size required for at least one of the methods to be effective was 200 in some conditions. When $\Delta \rho^{2} \geq .03$, a sample size of 50 to 100 was sufficient for at least one of the methods to be effective.

\section{Discussion}

We investigated coverage probability for the asymptotic CI and two percentile bootstrap CIs for $\Delta \rho^{2}$ in multiple linear regression analyses when predictors and criterion were normally distributed and $\Delta \rho^{2}$ described the strength of association for several predictors. We also investigated modified versions of these CIs. In general, the modified methods worked at least as well as their unmodified counterparts.

Specifically, results indicated that the traditional and modified bootstrap CI using $\Delta R^{2}$ performed poorly, except when $p-k=2$. Algina, et al. (2007) reported that when $\Delta \rho^{2}$ describes the strength of association for one predictor $(p-k=1)$, using the modified percentile bootstrap with $\Delta R^{2}$ to set a CI for $\Delta \rho^{2}$ resulted in good coverage probability in a wide range of conditions. Thus, the results, for the case in which $p-k=1$, do not generalize to the cases in which $\Delta \rho^{2}$ describes the strength of association for more than one predictor $(p-k>2)$.

The traditional and modified asymptotic CIs worked well in a variety of conditions. These results are contrary to results reported by Algina and Moulder (2001) who investigated the case of $p-k=1$ and reported that the traditional asymptotic CI tended to work poorly in many conditions with sample sizes of 200 or 


\begin{tabular}{|c|c|c|c|c|c|c|c|c|c|c|c|c|c|}
\hline \multirow[b]{3}{*}{$p$} & \multirow[b]{3}{*}{$p-k$} & \multirow{2}{*}{\multicolumn{4}{|c|}{$\begin{array}{c}\text { Modified } \\
\text { Bootstrap on } \Delta R^{2} \\
n\end{array}$}} & \multirow{2}{*}{\multicolumn{4}{|c|}{$\begin{array}{c}\text { Modified } \\
\text { Asymptotic } \\
n\end{array}$}} & \multirow{2}{*}{\multicolumn{4}{|c|}{$\begin{array}{c}\text { Modified } \\
\text { Bootstrap on } \Delta R_{c}^{2} \\
n\end{array}$}} \\
\hline & & & & & & & & & & & & & \\
\hline & & 50 & 100 & 150 & 200 & 50 & 100 & 150 & 200 & 50 & 100 & 150 & 200 \\
\hline 3 & 2 & 55 & $\underline{71}$ & $\underline{76}$ & 75 & 43 & $\underline{71}$ & $\underline{76}$ & 78 & 59 & 61 & 69 & 69 \\
\hline \multirow[t]{3}{*}{5} & 2 & 66 & 73 & 76 & 77 & 26 & 61 & 74 & 78 & 47 & 63 & 67 & 69 \\
\hline & 3 & 20 & 36 & 48 & 55 & 66 & 72 & 74 & 77 & 78 & 78 & 77 & 78 \\
\hline & 4 & 9 & 11 & 13 & 22 & 70 & 71 & 72 & 71 & 78 & 78 & 78 & 78 \\
\hline \multirow[t]{5}{*}{7} & 2 & 68 & 73 & 76 & $\underline{78}$ & 23 & 35 & 70 & $\underline{78}$ & 33 & 60 & 67 & 68 \\
\hline & 3 & 37 & 46 & 56 & 60 & 64 & 72 & 74 & 78 & 77 & 76 & 76 & 77 \\
\hline & 4 & 13 & 16 & 25 & 28 & 73 & 71 & 72 & 72 & 78 & 78 & 78 & 78 \\
\hline & 5 & 9 & 8 & 10 & 12 & 54 & 75 & 77 & 76 & 77 & 78 & 78 & 78 \\
\hline & 6 & 10 & 6 & 8 & 9 & 29 & 73 & $\underline{78}$ & $\underline{78}$ & 18 & 70 & $\underline{78}$ & $\underline{78}$ \\
\hline \multirow[t]{6}{*}{8} & 2 & 70 & 75 & 77 & 78 & 23 & 24 & 67 & 74 & 30 & 59 & 65 & 69 \\
\hline & 3 & 41 & 51 & 57 & 60 & 60 & 72 & 75 & 77 & 67 & 74 & 74 & 76 \\
\hline & 4 & 20 & 21 & 28 & 34 & 71 & 73 & 72 & 72 & 78 & 78 & 78 & 78 \\
\hline & 5 & 13 & 10 & 13 & 15 & 53 & 78 & 76 & 76 & 77 & 78 & 78 & 78 \\
\hline & 6 & 10 & 6 & 7 & 11 & 25 & 72 & 77 & $\underline{78}$ & 23 & 73 & 78 & $\underline{78}$ \\
\hline & 7 & 10 & 6 & 6 & 7 & 12 & 42 & 76 & 78 & 4 & 26 & 54 & 64 \\
\hline \multirow[t]{7}{*}{9} & 2 & 62 & 75 & 77 & 78 & 17 & 20 & 57 & 74 & 28 & 58 & 66 & 69 \\
\hline & 3 & 45 & 52 & 58 & 62 & 63 & 69 & 75 & 78 & 56 & 73 & 72 & 77 \\
\hline & 4 & 23 & 25 & 29 & 38 & 71 & 72 & 72 & 72 & 78 & 78 & 78 & 78 \\
\hline & 5 & 13 & 12 & 15 & 19 & 51 & 77 & 77 & 77 & 77 & 78 & 78 & 78 \\
\hline & 6 & 10 & 8 & 9 & 11 & 28 & 74 & $\underline{78}$ & $\underline{78}$ & 37 & 73 & $\underline{78}$ & $\underline{78}$ \\
\hline & 7 & 12 & 6 & 7 & 9 & 24 & 45 & 66 & 69 & 23 & 41 & 55 & 59 \\
\hline & 8 & 10 & 6 & 6 & 6 & 5 & 19 & 38 & 55 & 1 & 7 & 27 & 38 \\
\hline
\end{tabular}




\section{I. FOR SQUARED MULTIPLE SEMIPARTIAL CORRELATION}

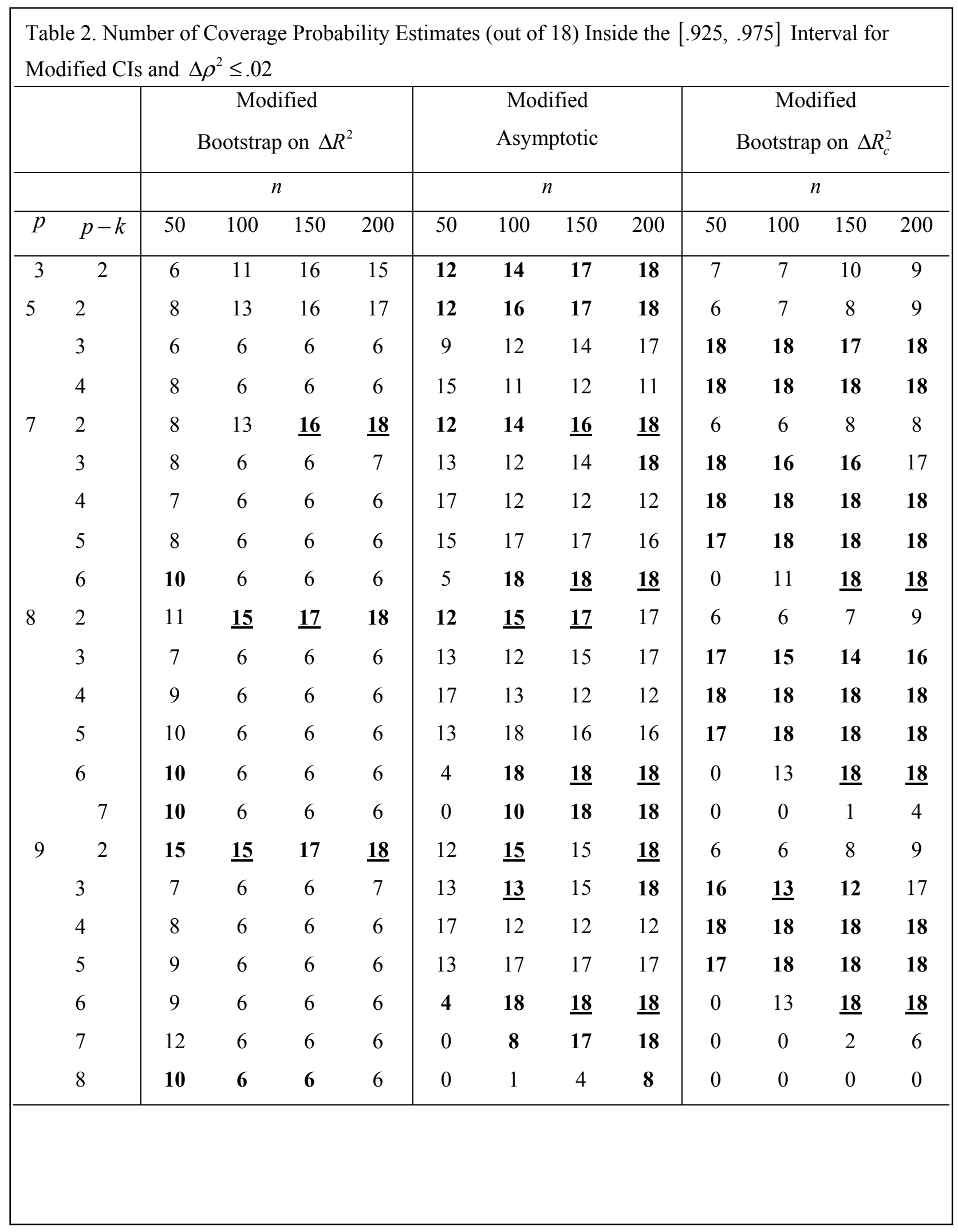




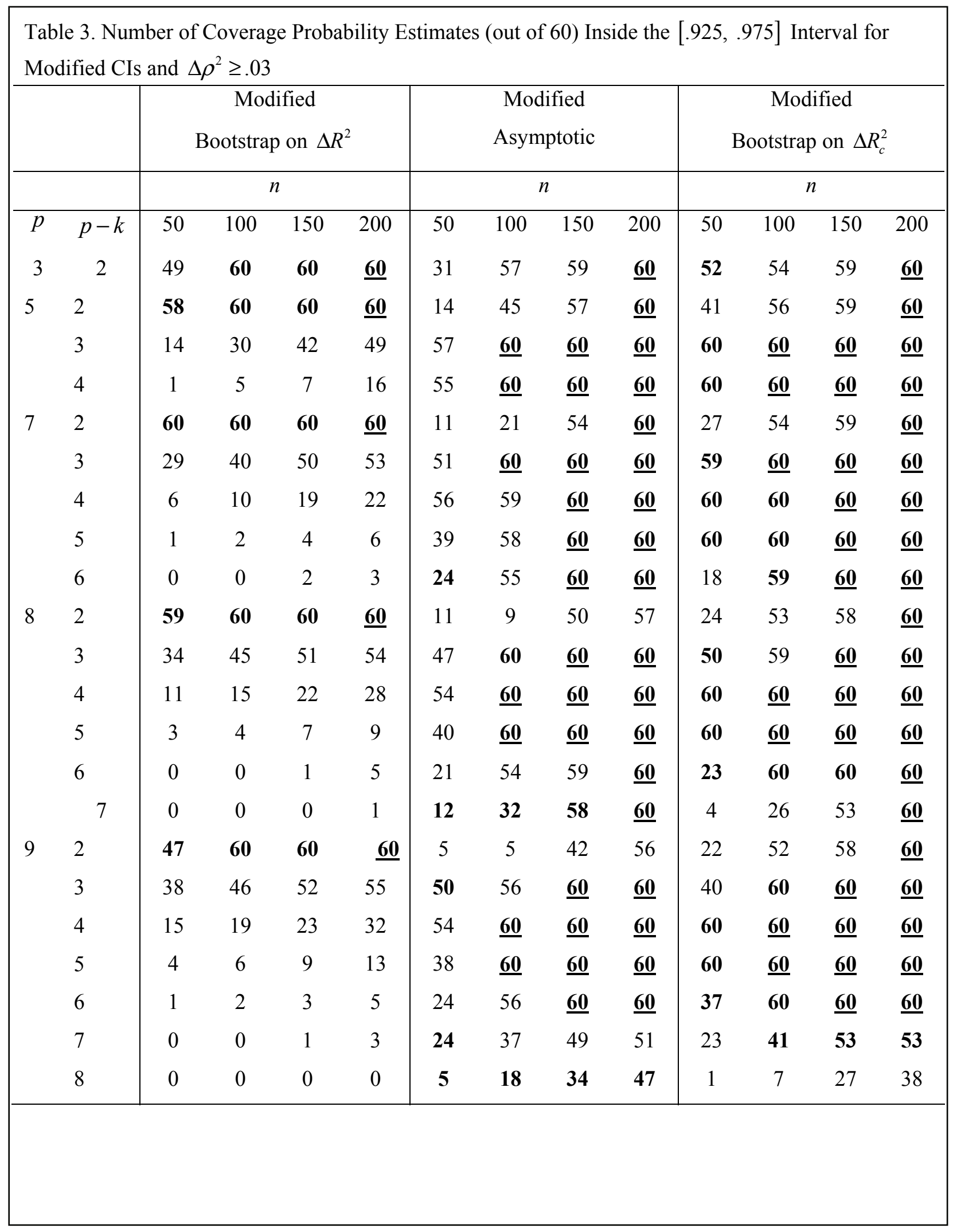




\section{I. FOR SQUARED MULTIPLE SEMIPARTIAL CORRELATION}

less. Algina and Moulder did not report results on the modified asymptotic CI, but the modification used in this paper is designed to improve performance when $\Delta \rho^{2}$ is zero, so that it is unlikely that using the modification with $p-k=1$ would overcome the problems that Algina and Moulder reported.

Unfortunately, although the modified asymptotic CI and the modified bootstrap CI on $\Delta R_{c}^{2} \quad$ worked better than the competitors investigated in this study, neither of these CIs worked well in all of the conditions we investigated. Particularly problematic was the condition in which the number of predictors in the full model was nine and the number of predictors in the reduced model was no larger than two. Defining adequate control of coverage probability by at least 77 of the 78 combinations of $\rho_{r}^{2}$ and $\rho_{f}^{2}$ for each combination of $p$ and predictors in the reduced model was no larger than two. Defining adequate control of coverage probability by at least 77 of the 78 combinations of $\rho_{r}^{2}$ and $\rho_{f}^{2}$ for each combination of $p$ and $p-k$, we offer the following recommendations for a CI method and sample size in order to achieve adequate control of coverage probability,

(a) If $p-k=2$ and $p \leq 7$, the modified asymptotic CI should be used with a sample size of at least 200. For $p \geq 8$, a sample size of 150 and the modified bootstrap CI using $\Delta R^{2}$ should be used;

(b) If $p-k=3$ and $p \leq 7$, the modified bootstrap CI using $\Delta R_{c}^{2}$ should be used with a sample size of at least 50. If $p \geq 8$ the modified asymptotic CI should be used with a sample size of at least 200;

(c) If $p-k=4$ or 5 and $p \leq 9$, the modified bootstrap CI using $\Delta R_{c}^{2}$ should be used with a sample size of at least 50 ;

(d) If $p-k=6$ (and therefore $p \geq 7$ ), the modified bootstrap CI using $\Delta R_{c}^{2}$ should be used with a sample size of at least 150 ;

(e) If $p-k \geq 7$ (and therefore $p \geq 8$ ), the modified asymptotic CI should be used and the sample size should 200 if $p=8$ and should be larger than 200 if $p=9$.

\section{References}

Alf, E. F., Jr., \& Graf, R. G. (1999). Asymptotic confidence limits for the difference between two squared multiple correlations: A simplified approach. Psychological Methods, 4, 70-75.

Algina, J., Keselman, H. J., \& Penfield, R. D. (2007). Confidence intervals for an effect size measure in multiple linear regression. Educational and Psychological Measurement, 67, 207-218.

Algina, J., \& Moulder, B. C. (2001). Sample sizes for confidence intervals on the increase in the squared multiple correlation coefficient. Educational and Psychological Measurement, 61, 633-649.

Browne, M. W. (1969). Precision of prediction (Research Bulletin 69-69). Princeton, NJ: Educational Testing Service.

Browne, M. W. (1975). Predictive validity of a linear regression equation. British Journal of Mathematical \& Statistical Psychology, 28, 79-87.

Cohen, J, Cohen, P, West, S. G., \& Aiken, L. S. (2003). Applied multiple regression and correlation for the behavioral sciences ( ${ }^{\text {rd }}$ ed.). Mahwah, NJ: Erlbaum.

Graf, R. G., \& Alf, E. F., Jr. (1999). Asymptotic confidence limits for partial and squared partial correlations. Applied Psychological Measurement, 23, 116-119.

Hedges, L. V., \& Olkin, I. (1981). The asymptotic distribution of commonality components. Psychometrika, 46, 331-336.

Mood, A. M. (1969). Macro-analysis of the American educational system. Operations Research, 17, 770-784.

Mood, A. M. (1971). Partitioning variance in multiple regression analyses as a tool for developing learning models. American Educational Research Journal, 8, 191-202.

Pedhazur, E. J. (1997). Multiple regression in behavioral research ( ${ }^{\text {rd }}$ ed.). Belmont. CA: Wadsworth.

Olkin, I., \& Finn, J. D. (1995). Correlations redux. Psychological Bulletin, 188, 155-164.

Stuart, A., Ord, J. K., \& Arnold, S. (1999). The advanced theory of statistics $\left(6^{\text {th }}\right.$ ed., Vol. $\left.2 \mathrm{~A}\right)$. New York: Oxford University Press.

Wilcox, R. R. (2003). Applying contemporary statistical techniques. San Diego: Academic Press. 